\title{
Tangence
}

\section{Un labo dans le poète : la fonction du discours scientifique dans les manifestes de Claude Péloquin}

\section{Jacques Paquin}

Numéro 61, décembre 1999

Savoir et littérature

URI : https://id.erudit.org/iderudit/008166ar

DOI : https://doi.org/10.7202/008166ar

Aller au sommaire du numéro

Éditeur(s)

Presses de l'Université du Québec

ISSN

0226-9554 (imprimé)

1710-0305 (numérique)

Découvrir la revue

Citer cet article

Paquin, J. (1999). Un labo dans le poète : la fonction du discours scientifique dans les manifestes de Claude Péloquin. Tangence, (61), 73-89.

https://doi.org/10.7202/008166ar d'utilisation que vous pouvez consulter en ligne. 


\title{
Un labo dans le poète: la fonction du discours scientifique dans les manifestes de Claude Péloquin
}

\author{
Jacques Paquin, \\ Université du Québec à Trois-Rivières
}

Plusieurs discours se combinent et s'enchevêtrent dans les manifestes de Péloquin: le discours hérité du surréalisme français et des automatistes québécois, le discours contre-culturel, qui fait l'apologie des hallucinogènes, le discours scientifique, décelable notamment dans le lexique utilisé et dans les démonstrations qui apparaissent à l'occasion sous forme de figures, enfin un discours que je qualifierais de technocrate dans la mesure où il s'appuie sur une gestion du savoir pour créer une société d'artistes-techniciens. Manifeste subsiste (1965), Manifeste Infra (1967) et Émissions parallèles (1967) formeront l'essentiel du corpus étudié. Comme l'œuvre de Péloquin n'a fait l'objet jusqu'ici d'aucun article de fond, je crois utile d'en dresser un bref bilan, d'autant plus que sa réception reflète assez bien le malaise de la critique devant les ambitions scientistes d'une écriture marquée par le surréalisme.

\section{Une œuvre controversée}

Le premier article consacré à Claude Péloquin paraît sous la plume de Jean Marcel, et porte sur Jéricho, publié pour la première fois en 1963 aux Publications Alouette ${ }^{1}$. Il s'agit d'un compte rendu peu exhaustif mais qui demeure bienveillant malgré les réserves du critique. Le principal reproche que Marcel adresse à Péloquin c'est de se servir de la poésie comme d'“une tribune ou un prétoire». Et il ajoute: "La poésie [...] si elle juge le monde c'est avec un droit qui n'a rien à voir avec le code civil. " 2 Mais des critiques plus substantielles et plus sévères accompagneront la parution de Manifeste subsiste (1965) et les recueils de poèmes Les mondes assujettis (1965) et Calorifère (1966). Max Laroche, qui

1. Jean Marcel, "Les trompettes de Jéricho, par Claude Péloquin ", L'Action nationale, vol. LIV, $\mathrm{n}^{\circ} 3$, novembre 1964, p. 278-279.

2. Ibid., p. 270, pour les deux citations. 
74

signe le compte rendu critique des deux premiers textes cités, y tient à peu près le même discours ; il raille "la logique subsiste" ("Si ce n'est pas la logique avec un grand L c'est peut-être d'une logique subsiste qu'il s'agit "3), alors que Guy Robert, pour sa part, déclare forfait devant "cette poétique abracadabrante" ${ }^{4}$.

Jusqu'à la parution du Manifeste Infra suivi des Émissions parallèles, publié à l'Hexagone, la critique institutionnelle demeure donc soit condescendante, soit ouvertement méprisante. La consécration viendra pourtant, en 1966, à l'occasion d'un entretien entre Paul Chamberland et Claude Péloquin, dans les pages de Parti pris $^{5}$. En outre, la revue ouvre ses pages à la publication de poèmes et d'extraits du manifeste. Le fait est remarquable puisque Chamberland n'a signé que deux articles littéraires dans Parti pris. C'est aussi lui qui fera le compte rendu critique du recueil Calorifère $^{6}$. "Fondation du territoire", l'un des articles majeurs sur la poésie québécoise de l'époque (1967), place Claude Péloquin comme le point d'arrivée de toute l'histoire de la poésie depuis Alain Grandbois, en l'excluant toutefois du "champ thématique de la fondation" ${ }^{7}$. Enfin, toujours dans la même revue, Patrick Straram, une fois, cède entièrement la parole à Claude Péloquin dans sa chronique "Interprétation de la vie quotidienne" ${ }^{8}$. Toujours en 1967, la revue Etudes françaises invite Péloquin à livrer un témoignage sur Nelligan, ce qui montre assez la considération que Péloquin commence à recevoir de la part de l'institution ${ }^{9}$.

Les années 1969 et 1970 seront particulièrement florissantes pour le poète. Autant on parlera de lui, autant on lui donnera l'occasion de proposer sa conception de la poésie. Outre la place importante que lui a réservée Paul Chamberland dans l'histoire de

3. "Manifeste subsiste de Claude Péloquin", Livres et auteurs canadiens 1965, Montréal, Éditions Jumonville, 1966, p. 83.

4. "Calorifère de Claude Péloquin ", Livres et auteurs canadiens 1965, Montréal, Éditions Jumonville, 1966, p. 92.

5. Paul Chamberland, "Faire le voyage. Entretien avec Claude Péloquin", Parti pris, vol. III, avril 1966, p. 38-45.

6. Paul Chamberland, "Nous ne sommes pas au monde: Giguère, Péloquin", Parti pris, vol. III, no 7, février 1966, p. 59-61.

7. Paul Chamberland, "Fondation du territoire", Parti pris, vol. IV, n ${ }^{\text {os }}$ 9-12, maiaoût 1967, p. 42.

8. "Long distance call minuit vrai", Parti pris, vol. IV, $\mathrm{n}^{\mathrm{os}}$ 5-6, janvier-février 1967, p. 101-103.

9. "Témoignages d'écrivains", Études françaises, vol. III, n 3, 1967, p. 301-307. 
la poésie, la présence publique de Péloquin — par le biais de spectacles, l'écriture de chansons et surtout, par sa participation à la Nuit de la poésie 70 - lui donnera une visibilité extraordinaire. Ainsi Pierre de Grandpré fait mention du poète à quelques reprises et le présente comme l'un des représentants de la poésie québécoise contemporaine et comme produit d'une "poésie [qui] se fait d'autre part de plus en plus "magique" en plusieurs sens, dont celui de la pullulation des images qu'elle engendre: CLAUDE PÉLOQUIN surtout [...]"10. Gilles Marcotte dans Le temps des poètes glisse rapidement sur son nom, mais pour le relier au "postsurréalisme" "11. Cécile Cloutier fait appel à sa conception de la poésie dans sa vaste enquête auprès des poètes ${ }^{12}$ et Clément Moisan l'identifie au concrétisme dans L'âge de la littérature canadienne ${ }^{13}$. La presse elle-même s'intéresse au phénomène Péloquin: si le chroniqueur du Devoir y fait rapidement allusion ${ }^{14}$, la revue Perspectives lui consacre deux portraits, l'un sous forme d'autoportrait, l'autre sous forme d'entrevue avec Denise Boucher ${ }^{15}$. À cette époque, les contributions de Péloquin aux chansons de Charlebois sont connues (Lindberg et CPR Blues), ce qui l'insère, bien que marginalement, dans le circuit des vedettes du spectacle. Mais c'est sa participation à La Nuit de la poésie 1970 qui le réconciliera avec une partie de l'institution littéraire. Guy Robert qui, on se le rappelle, était resté plutôt tiède devant ses premiers recueils, montre un enthousiasme sans réserve en le comptant parmi ses camarades, au même titre que son ami le peintre Albert Dumouchel. Il pousse l'audace jusqu'à faire trôner Péloquin parmi "les plaideurs les plus passionnés et vertigineux de la "grande

10. Pierre de Grandpré et al., Histoire de la littérature française du Québec, tome III (1945 à nos jours), Montréal, Librairie Beauchemin, 1969, p. 282.

11. Gilles Marcotte, Le temps des poètes, HMH, Montréal, 1969, p. 223.

12. Cécile Cloutier-Wojciechowska, "Témoignages des poètes canadiens-français. Enquête littéraire", dans Paul Wycynski et al., La poésie canadiennefrançaise, Montréal, Fides, 1969, p. 584-585. La même année, elle l'intègre à un article consacré à "La poésie contemporaine au Québec" (Revue d'esthétique, vol. XXII, no 3, juillet-septembre 1969, p. 236-250).

13. Clément Moisan, L'âge de la littérature canadienne, Montréal, HMH, coll. "Constantes", 1969, p. 157-158.

14. Jean Basile, "Au Québec, il y a au moins 4000 poètes", Le Devoir, vol. LXI, $\mathrm{n}^{\circ} 73,30$ mars 1970, p. 8.

15. Dans l'ordre: "Claude Péloquin., écrivain (1970)", Perspectives, vol. XII, $n^{\circ}$ 50, 12 décembre 1970, p. 29; Denise Boucher, "Jean-Claude Péloquin, alias Pélo, poète de chez nous: J'aime l'homme, le Québec, la bière et je déteste la mort", Perspectives, vol. IX, n 41, 11 octobre 1969, p. 25-29 et 31. 
76

Cause de la mort de la mort", comme les Russes Tolstoï dans La mort d'Ivan Illich, ou Soljenitsyne dans Le pavillon des cancéreux". Ce revirement a pour origine la prestation de Claude Péloquin lors de la Nuit de la poésie 1970. Le même critique écrira, à ce propos " [que la Nuit] a trouvé bien au-delà des discours de circonstances [...] son point de convergence et de vertige dans la présence stone mais combien bouleversante de Pélo" ${ }^{16}$. La même année, au Grand Théâtre de Québec, Jordi Bonet gravait sur une muraille les vers désormais célèbres "Vous êtes PAS écoeurés de mourir / bandes de caves / c'est assez!" qui ont lui assuré une notoriété, par leur ton de provocation, auprès d'un public plus large. L'œuvre de Péloquin peut donc d'ores et déjà être considérée comme faisant partie intégrante de la poésie contemporaine québécoise ${ }^{17}$.

Le premier manifeste, Manifeste subsiste ${ }^{18}$, comporte huit parties, précédées d'une "Préface". Bien que le texte délivre un discours enthymématique, caractéristique du genre, les intitulés des parties suggèrent davantage l'idée d'une étude; dans "Note", par exemple, les divisions sont qualifiées de "chapitres" (Subsiste, p. 135). Dans la partie intitulée "Position et position", qui rappelle les "Positions et propositions" claudéliennes, l'accent est mis

16. Guy Robert, "Chroniques: la nuit blanche des poètes", Liberté, vol. XII, $\mathrm{n}^{\circ} 2$, mars-avril 1970, p. 117-118. Même son de cloche chez Jean Royer pour qui "l'un des grands moments de cette nuit fut celui de Claude Péloquin" ("Pour le bilan d'une nuit québécoise ", L'Action, 4 avril 1970, p. 18.)

17. Pierre Nepveu et Laurent Mailhot, dans la réédition de leur anthologie ( $L a$ poésie québécoise des origines à nos jours, Montréal, Typo, 1986) se contentent de faire mention de son manifeste ("Claude Péloquin fait un éloge enthousiaste de la modernité et du surréalisme dans son Manifeste infra", p. 29), sans pour autant daigner l'inclure parmi les poètes retenus. Par ailleurs, Clément Moisan va non seulement clore son histoire de la poésie contemporaine avec Péloquin, mais encore va-t-il extraire une définition de la poésie moderne: "Claude Péloquin, qu'il faudrait aussi ne pas laisser de côté, précise peut-être le but, avoué ou non, de ces poétiques diverses [...]. $\mathrm{Au}$ fond, il s'agit encore, après Rimbaud souvent cité par ces poètes, de changer la vie. Et pour cela, "pénétrer l'inconnu". Après quoi, une bonne définition de cette poésie serait celle de Péloquin: La poésie c'est ce qui crée l'impossibilité / magnifique de ne pas écrire" (cf. "La littérature québécoise contemporaine 1960-1977 I. La poésie", p. 300). La citation de Péloquin est tirée de Éternellement vôtre, Éd. du Jour, 1974, p. 124.

18. Manifeste subsiste dans Les mers détroublées. Poèmes et textes: 1963-1969, tome 1, Montréal, Guernica, 1993. Ce texte est paru pour la première fois à compte d'auteur en 1965, puis repris dans Le premier tiers, volume II, chez Beauchemin, 1976. Les références ultérieures seront indiquées entre parenthèses dans le texte, à partir de l'édition de 1993. 
sur le caractère insolite de l'Art et sur la transgression de l'Interdit, en vue d'accéder à un univers qui porte le nom de "l'Arrièrechose", désigné en termes antithétiques, par le biais d'une image: "[...] là où tous les systèmes se noient sans eau" (Subsiste, p. 136). Péloquin rejoint les surréalistes, pour lesquels l'imagination constituait en elle-même une réalité plus vraie que le réel qui s'offrait aux sens. C'est à une exaltation de l'irrationalité et de l'illogisme que convie le texte du Manifeste subsiste:

On ne possède plus, on n'analyse plus l'aventure, on s'envole avec, un point c'est tout. La véritable aventure poétique est celle qui ne se permet plus d'escale, celle qui se veut amalgamée à un songe sans retour; là réside l'espoir de voir l'homme délivré du superflu. C'est la seule alternative pour celui-ci de vivre Ailleurs (ici) et, libéré, de tenir debout par de constants sabotages des tabous. (Ibid., p. 140)

Où se trouve cet Ailleurs-ici? Il est dans l'homme cosmique, c'està-dire à la fois dans l'univers (le Cosmos) et à l'intérieur de l'homme. Voilà, en substance, l'essentiel du propos qui sera développé dans les trois manifestes. Le Manifeste subsiste affiche une nette inclination pour le surréalisme tandis que les manifestes Infra et Émissions parallèles font porter leur discours sur l'expérimentation de voyages hallucinatoires et sur l'importance de les inscrire au sein d'une science à caractère expérimental.

Tous ces textes ont comme origine une pratique du spectacle qui a pour souci d'intégrer plusieurs disciplines. Ainsi est né le groupe des Horlogers qui deviendra par la suite Le Zirmate et dont certains membres seront les signataires du manifeste Infra, identifiés chacun selon sa discipline respective. Outre Péloquin, poète et rédacteur du manifeste, on trouve les noms de Serge Lemoyne (peintre), Pierre Cornellier (peintre), Jean Sauvageau (musicien) et Gilbert Labelle (mathématicien). Le Zirmate se définit comme "un groupe de recherche dans l'expression de l'insolite, du caché et de la phénoménologie du fantastique dans le subconscient et ses univers environnants" (Infra, p. 178). La filiation avec les surréalistes est ouvertement reconnue par Serge Lemoyne, qui confie, dans un entretien: "Nous respectons énormément Borduas; dans son ouvre, il y a ruptures, il n'a pas craint d'aller ailleurs, de toujours recommencer. Nous, on vou-

19. Cité dans André-G. Bourassa, Surréalisme et littérature québécoise, Montréal, Les Herbes rouges, 1986, coll. "Typo", p. 462. L'origine de cette citation se 
drait être un peu comme ça." ${ }^{19}$ Par ailleurs, le groupe tente de se démarquer de cette influence de diverses manières. Déjà, dans le Manifeste subsiste, on visait à la conciliation plutôt qu'à la confrontation, et on prenait ses distances par rapport à la violence gratuite préconisée par les surréalistes français: "Il ne suffit plus de fondre sur une foule avec une arme pour faire acte subsisto-surréaliste, encore faut-il payer à boire ensuite" (Subsiste, p. 137). Dans son entrevue avec Chamberland, Péloquin affirme avoir quitté "le côté révolte du surréalisme" 20 . D'un côté, le manifeste Subsiste dénonce, il identifie même ses opposants, de l'autre, il recherche un terrain neutre à partir duquel il pourrait réconcilier tous les mouvements antagonistes ${ }^{21}$. Le manifeste veut se distinguer de ses prédécesseurs dans la mesure où la poétique qui est proposée s'appuie sur la notion de recherche: "Nous marquons un pas de plus par rapport au Refus global: nous pouvons avoir les laboratoires, entreprendre un travail plus rigoureux." ${ }^{22}$ Cette recherche est indissociable du voyage, qui est au cour de la démarche: "Ce n'est pas tout d'écrire, encore fautil faire le voyage préalablement avec les symboles; il ne s'agit donc plus d'automatisme" (Infra, p.29). Le manifeste Infra marque un pas de plus dans l'affranchissement du discours automatiste, jugé inapte à s'insérer dans l'ère technologique. La justification d'une telle distanciation? "Le résultat doit être connu à l'avance et mis au point avec les instruments du langage. Voilà pourquoi Infra parle de voyages au préalable." (Émissions parallèles, p. $48^{23}$ ). On considère que Claude Gauvreau est "un poète plutôt mystique (vie intérieure)" ${ }^{24}$, alors que ce n'est pas l'automatisme que valorise Péloquin mais les symboles. Par

trouve dans Gil Courtemanche, "Nouvel Age, Nouvel Art, vieux problème?", La presse, 18 avril 1964.

20. Paul Chamberland, "Faire le voyage. Entretien avec Claude Péloquin", loc. cit., p. 41.

21. Mais la tendance du genre est la plus forte et ne peut empêcher le manifeste d'énumérer, comme il se doit, une liste noire des ennemis de la poétique subsiste.

22. Paul Chamberland, "Faire le voyage. Entretien avec Claude Péloquin", loc. cit., p. 42.

23. Infra recouvre tout aussi bien la philosophie mise de l'avant que l'énonciateur, c'est pourquoi on retrouve cette dénomination dans les Émissions parallèles.

24. Paul Chamberland, "Faire le voyage. Entretien avec Claude Péloquin", loc. cit., p. 45. 
symboles, il entend ceux qui proviennent des langages mathématiques et de la technologie. Parallèlement aux réserves qu'il exprime envers Gauvreau, il refuse d'entériner l'influence d'Antonin Artaud mais choisit plutôt Michaux 25. C'est très probablement au Michaux adepte des expériences avec les drogues que songe Péloquin. Un extrait tiré de Plume pourrait éventuellement constituer l'intertexte d'une bonne part du discours de Claude Péloquin: "J'aurais pourtant voulu être un bon chef de laboratoire, et passer pour avoir bien géré mon "moi" ". 26

D'autre part, la reconnaissance tacite de la tradition issue de la génération des années d'après-guerre entraîne les attaques contre la génération de l'Hexagone, qu'on estime trop centrée sur l'expression lyrique et sur la figure du pays; on s'en prend même à Parti pris, qui défend la fonction sociale de la poésie. Les assauts contre l'un ou l'autre sont virulents, notamment dans le manifeste Infra, pourtant publié à l'Hexagone: “Ici, la poésie s'est arrêtée aux mouettes à n'en plus finir, aux sentiments examinés au microscope, à une certaine nostalgie de soir d'hiver et à une attitude révolutionnaire qui n'est pas de son ressort. " ${ }^{27}$ La divergence entre la conception de la poésie des aînés et celle de Péloquin est clairement mise en valeur un peu plus loin: "[...] poétiquement, la femme, un pays, des beaux sentiments ou quatre saisons n'ont rien de comparable avec la folie, l'asphalte ou la mathématique" (Infra, p. 19-20). Péloquin use d'un discours qui s'écarte soit de manière intrinsèque, soit de manière stratégique, du discours de fondation ou de celui de l'Hexagone. À Chamberland qui lui demande si pour lui la poésie est communication ou expression, Péloquin répond: "La poésie a pour but l'émission de dimensions nouvelles. Il s'agit d'émettre." 28 Comme l'a souligné Jean-Louis Major, les manifestes de Péloquin tracent une voie nouvelle en rompant avec l'esprit de la génération

25. Ibid., p. 43. On notera par ailleurs que Péloquin ne réfère jamais aux autres poètes qui pratiquaient l'automatisme, comme Giguère ou Hénault, limitant ses influences au groupe du Refus global.

26. Plume, précédé de Lointain intérieur, Paris, Gallimard (c1963), 1996, p. 216. Cet extrait est tiré de la "Postface".

27. Manifeste Infra suivi des Émissions parallèles, Montréal, l'Hexagone, (c1967) 1974, p. 19. Ces textes ont été repris, avec des modifications, dans Le premier tiers, volume 1, chez Beauchemin, 1976, ainsi que chez Guernica, Montréal, sous le titre Les mers détroublées. Poèmes et textes: 1963-1969, tome 1, 1993. Toutes les références ultérieures à l'un ou l'autre de ces manifestes renverront à l'édition de 1974 (l'Hexagone), avec simple mention de page.

28. "Faire le voyage. Entretien avec Claude Péloquin", loc. cit., p. 42. 
80

de l'Hexagone mais en maintenant la continuité avec l'automatisme québécois d'après-guerre: "Historiquement, l'Hexagone pourrait se situer entre deux manifestes: Refus global (1948) [...] le Manifeste subsiste (1965) qui, sans avoir (et de très loin) l'importance de Refus global, indique peut-être malgré tout de nouvelles orientations en poésie québécoise et marque un écart symptomatique par rapport à l'esprit de la poésie 1950-1965." 29

\section{Des désirs esthétiques aux délires psychédéliques}

Surréalisme et automatisme débouchent, chez Péloquin, sur un discours de la contre-culture. Le nom de Michaux pourra servir d'amorce pour aborder cette question. La présence de Michaux n'est pas si incongrue qu'elle apparaît au départ. Dans l'entourage du surréalisme, sans y être jamais totalement lié, par souci d'indépendance, Michaux s'est livré lui aussi à des expériences sur le moi, par le biais de stupéfiants, mais toujours sous la supervision d'un médecin et de témoins de ce qui constituait pour lui une véritable expérience au sens scientifique. D'autre part, son intérêt pour les autres cultures, comme celle des mystiques de l'Orient, en fait l'un des précurseurs d'un certain esprit de la contre-culture ${ }^{30}$. Le courant surréaliste et la contreculture font ainsi bon ménage notamment par le biais de la marque du discours délirant, dont est friand le manifeste ${ }^{31}$. C'est particulièrement dans le caractère fortement individuel de leur conception du politique que se rejoindraient surréalisme et contre-culture, selon Gaétan Rochon :

[Le surréalisme] a essayé de concilier le changement mental et le changement social, de jeter un pont entre la révolution à l'état pur, individuelle, un peu abstraite, encore près de la révolte et la pratique politique de la révolution réelle. L'histoire de ses rapports avec le communisme n'est pas sans rappeler ceux de la contre-culture avec la politique. ${ }^{32}$

Rochon fait référence aux activistes de la contre-culture, auxquels

29. J.-L. Major, "L'Hexagone: une aventure en poésie" dans La poésie canadiennefrançaise, sous la dir. de Paul Wycynski et al., Montréal, Fides, 1969, p. 182.

30. Gaétan Rochon, Politique et contre-culture. Essai d'analyse interprétative, Montréal, Cahiers du Québec/Hurtubise HMH, 1979, p. 63.

31. Cf. Wladimir Krysinski, "Une automobile, une mitraillette, une gifle et un singe crevé: Marinetti et ses avatars slaves", Études françaises, vol. XVI,

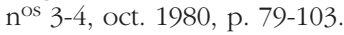


on ne peut identifier entièrement Péloquin, plus anarchiste que militant. Fait à noter, la contre-culture de Péloquin se nourrit en majeure partie de sources européennes. Or, il semble que la contre-culture n'ait fait que trop peu de cas de sa propre histoire et se soit contentée de relier cette dernière à ses manifestations américaines. Pourtant, certains ont voulu voir dans les mouvements dadaïstes et surréalistes l'origine du mouvement ${ }^{33}$. C'est effectivement chez ces derniers que Péloquin puise le recours à l'insolite, alors que les références aux représentants de la contreculture sont à peu près nulles. La contestation de la culture officielle se traduit chez Péloquin par des rapports ambigus: d'un côté, il semble bien qu'il ait été formé à une école héritière de l'enseignement classique - les parodies argumentatives que constituent les manifestes le montrent assez ${ }^{34}$ - mais plus important encore, Péloquin nie que les noms de Artaud, Breton et Michaux, qui apparaissent à quelques reprises sous sa plume, aient eu quelque influence déterminante. Inféodé à une contreculture "montante", Péloquin ne s'affiche pas comme un grand lecteur et, s'il cite telle figure de la littérature française, c'est pour aussitôt écarter du revers de la main le poids de l'histoire en général et de l'histoire culturelle en particulier. Il écrit dans son autobiographie: "En deux jours deux textes - l'un d'Artaud et l'autre de Huxley. Ce qui me chatouille c'est qu'on pensera qu'ils m'ont influencé. Ils le font dans une certaine mesure certes mais après que je l'aie écrit- car au cours des années je n'ai jamais lu mais bien plutôt feuilleté les grands esprits. " 35 Clément Moisan prélève cette affirmation provocante de Péloquin: "C’est vrai qu'on est des sauvages - Et après? Il nous reste au moins ça!»36 Le choix des mots constitue d'ailleurs une contrainte à

32. Gaétan Rochon, op. cit., p. 65.

33. Gaétan Rochon, op. cit., p. 63. Voir aussi la note 10, p. 180.

34. Si les textes de Péloquin ne font nullement référence à une culture scientifique reconnue, il reste que ses vagues allusions à l'arithmétique, à l'astronomie et à la géométrie peuvent rappeler les sciences que Platon, dans $L a$ République (livre VII), range parmi celles qui doivent servir à la formation des philosophes. Platon retient également la musique et la dialectique.

35. Une plongée dans mon essentiel, Montréal, Guernica, 1985, p. 83.

36. Cité par Clément Moisan, "La littérature québécoise contemporaine 19601977", Etudes françaises, octobre 1977, vol. XIII, n ${ }^{\text {os } 3-4, ~ p . ~ 279 . ~ L a ~ c i t a t i o n ~}$ provient d'Éternellement vôtre, op. cit., p. 19.

37. Ce texte, qui combine prose et vers, fait partie de la réédition intitulée Les mers détroublées, déjà donnée en référence. On lit, à la suite de l'expression "vaches homogénéisées", cette note infrapaginale qui en dit long sur les 
82

laquelle refuse de se soumettre l'auteur de Pour la grandeur de l'homme ${ }^{37}$. La culture est affichée, mais avec réticence, puisqu'on l'accuse d'être lacunaire ou trompeuse. Or, les manifestes s'engagent dans un cheminement qui, sous plusieurs aspects, sont incompatibles avec le discours idéologique de la nouvelle culture qui aura cours dans les années soixante-dix. Le fondement de la critique contre-culturelle repose sur la contestation de l'organisation technocratique de la société, et avance l'idée selon laquelle le développement de la science et des technologies, qui a engendré la société industrielle, finit par atomiser l'individu. En s'appuyant sur le discours de la revue Mainmise, Jules Duchastel a résumé les positions des tenants de la contre-culture par rapport à la place de l'homme:

Un des thèmes majeurs de la revue est de prôner le retour aux facultés et aux capacités inscrites en l'homme. L'homme est perçu comme étant dénaturé. Il faut donc travailler à le retrouver. Quelles sont ces capacités perdues? Ce sont la créativité, l'émancipation de la personnalité, l'autonomie, l'exercice du libre choix et de l'initiative. ${ }^{38}$

Le manifeste Subsiste dispose les principaux jalons de la croyance en l'exploration de l'inconnu: "Il est possible maintenant de créer des mythes nouveaux parce que les symboles de l'Ailleurs nous sont de plus en plus connus." (p. 137). Les textes suivants vont préciser le programme tout en plaçant la libération et la transformation de l'homme au sein de leurs préoccupations. "La seule survie de l'homme, lit-on en conclusion des Émissions parallèles, réside dans la croyance illimitée de ses pouvoirs, illimités pour longtemps encore" (p. 45); ce qui fait écho à la conclusion du manifeste Infra publié conjointement: "Les espaces nouveaux que s'ouvre l'homme vont le faire éclater s'il ne sait pas faire la

rapports que Péloquin entretient avec la langue française: "À chaque fois que je trouve le dictionnaire je me sens ridicule. C'est la dernière fois. Je vais désormais écrire et publier le français le plus compréhensible possible, Madame la langue française, je vous ai dans le cul." (p. 314). Plus loin, l'auteur, au moment de la révision de son texte, rédige le commentaire suivant: "j'ai appris il y a quelques années après la parution de la première édition du présent ouvrage que Touareg était le pluriel de Targi. Fuck, ça va rester de même!" (p. 322)

38. Jules Duchastel, "La contre-culture: l'exemple de Mainmise", dans L'avantgarde culturelle et littéraire des années 70 au Québec, sous la dir. de Jacques Pelletier, Montréal, 1986, Université du Québec à Montréal, p. 65, "Les Cahiers du département d'études littéraires ", n 5 . 
Lumière dans les Dedans du Cosmos...." (p. 37). Prépondérance de l'individu sur la collectivité, libération des facultés créatrices individuelles (à commencer par le désir sexuel et par l'expérimentation de drogues qui favorisent l'élargissement de la conscience de soi), désir de transformation de l'homme par l'intérieur, valorisation d'attitudes déviantes (comme la folie) ont été revendiqués aussi bien par les surréalistes que par la génération qui donnera naissance aux mouvements des années soixante-dix. Là où les propos de Péloquin tranchent avec l'idéologie contreculturelle, c'est dans les moyens choisis pour réaliser la libération de l'individu. Le manifeste Infra s'ouvre avec la dédicace suivante: "À la N.A.S.A. /à tout ce qui est recherche /Et à la grandeur du sexe". Tandis que les deuxième et troisième vers sont tout à fait dans l'esprit de la contre-culture, le premier ("À la N.A.S.A.") ne laisse pas d'étonner dans la mesure où la liberté de l'individu doit passer par la reconnaissance de l'un des symboles les plus puissants du développement technologique, issu de l'impérialisme américain. Péloquin semble vouloir réaliser la quadrature du cercle en rattachant la survie de l'homme au développement des technologies d'avant-garde. L'évocation de la NASA se fonde sans doute sur l'analogie avec les voyages intérieurs, par l'intermédiaire de psychotropes. Une injonction comme "Il faut avoir la force d'halluciner et de s'halluciner à froid et lucidement " témoigne de la prédominance de l'espace intérieur dans le discours des manifestes. De même, l'évocation des sciences paranormales laisse à penser que les voyages intersidéraux sont tout au plus une métaphore commode pour rendre compte d'un voyage qui doit se dérouler à l'intérieur de l'homme, et dont l'artiste est le principal initié. La croyance aux sciences occultes est d'ailleurs une condition de reconnaissance: "est mort pour Infra celui qui est resté à la surface des univers existants ; par exemple, celui qui ne croit pas à la parapsychologie ou qui ignore l'existence du paranormal en tant que réalité pure." (Infra, p. 24). Un peu plus loin, on insiste sur la différence entre l'acceptation habituelle du voyage et la nouvelle signification que doit revêtir ce vocable: "Partir" ou "voyager", tels qu'entendus plus généralement, sont en dehors de la liberté Infra. Cette dernière réside dans le pouvoir d'imbriquer le réel avec Lui-Même, ce qui implique toutes ses facettes; c'est-à-dire, tant dans l'esprit cosmique que dans le corps cosmique." (Infra, p. 25). On l'aura compris, l'espace sidéral envisagé dans les manifestes est tout intérieur, comme le voyage. Aussi, peu importe que Péloquin uti- 
84

lise des termes comme "univers", "galaxies", "corps", force est de constater que "[l']engagement du poète est dans la recherche de zones intimes et toujours déchiffrables, tant dans l'homme qu'en ses univers" (Infra, p. 26). En somme, il s'agit surtout de provoquer la "libération de l'homme dans son esprit" (Ibid.).

\section{La science subjectivée}

Tandis que le premier manifeste de Péloquin, fortement imprégné de surréalisme, demeure relativement discret sur la question de la science et de la recherche, cet axe occupe une place stratégique dans le déploiement des arguments des manifestes suivants. Dans le Manifeste subsiste, c'est la valorisation de l'insolite, aspect essentiel de l'esthétique surréaliste, qui forme le noyau du discours. La recherche prise en elle-même se limite à inviter la société à rendre la consommation de l'art accessible à tous: "J'exige des fouilles systématiques dans les bibliothèques des communautés avares, l'exposition intégrale de toutes les œuvres d'art qui dorment dans ces consacrés lieux saints" (Subsiste, p. 147). Le manifeste se termine d'ailleurs sur une forme de retournement par le biais du rire, arme suprême qui rappelle Bataille et qui semble permettre, comme par une opération magique, le dégagement de toutes les contraintes rationnelles: "Entre deux éclats de rire / Ils [sic] s'affaissa d'une vision d'éternité." (Ibid., p.148). Les plus grandes occurrences du lexique scientifique reposent en majeure partie sur les mots expérimentation et recherche. Il reste que la science des manifestes demeure tributaire des positions surréalistes et contre-culturelles, elle privilégie l'intériorité de l'homme comme objet, alors que la science, telle qu'on la connaît, s'est plutôt attachée à délivrer un savoir qui porte sur les choses, par conséquent sur des objets extérieurs. Ici, l'extérieur est compris comme un microcosme qui se trouve à l'intérieur de l'homme, comme en faisaient état les anciennes épistémès. Gaétan Rochon explique en ces termes les conséquences d'une telle vision:

Avec sa méfiance à l'égard de la science, sa dénonciation des exploits techniques, l'exploration spatiale par exemple (aux États-Unis surtout et à ses débuts), sa définition des relations de

39. G. Rochon, op. cit., p. 28. 
l'homme avec la Nature, la contre-culture se trouve à renouer avec l'ancienne conception cosmogonique: la Terre est le centre de l'univers, l'Homme le roi de la création. 39

Par certains côtés, la philosophie des manifestes reste donc tributaire de l'idéologie de la contre-culture, mis à part le fait que la science et la technologie sont jugées parties prenantes du développement de l'individu, ainsi que de la littérature et des arts. Au demeurant, la science sert aussi de légitimation à l'idéologie contre-culturelle. On a recours à la science à partir de ses paradigmes les plus communs et les plus vagues, comme l'expérience, le laboratoire, la recherche, pour justifier une activité que la pensée rationaliste estime illogique et indigne de la démarche scientifique. Le recours à la science se traduit chez Péloquin par un recours stratégique pour la légitimation d'une pensée qui valorise l'illogisme et le précognitif :

Il n'y a pas que ce qui est dans la tradition de la logique et du dualisme qui soit civilisé; pas plus que ce qui est issu de la connaissance acquise et du prouvé. Partout sur cette planète-ci, il y a de ces "fous" qui travaillent sur l'Infra-structure de la Réalité dans des domaines que des esprits morts considèrent comme étant abusifs, chimériques et inutiles. (Infra, p. 15).

Cette stratégie vise donc, par le biais de l'expérimentation de l'homme sur lui-même, à "rendre logique ce qui aura été qualifié de folie pure" (Ibid.). Le savoir scientifique reste lié presque exclusivement au lexique utilisé, bien que Péloquin semble accorder beaucoup d'importance à ce qu'il dénomme la "dynamique-statique", notion sur laquelle il ménage de longs développements en truffant son discours de pseudo-formules mathématiques: "Jusqu'ici, les théoriciens avaient situé la statique d'une part et la dynamique à l'autre extrême... Je suis positif que [sic] l'interfonte des deux donne la dimension exacte où se situe l'Éveil... Dans le $-1,0$ et le plus $1 \ldots$ Tous les trois étant au même niveau et de la même densité dans un signe plus (+), sans fin" (Émissions parallèles, p. 64). Quelques pages plus loin, l'auteur appuie son discours sur la notion de "courbe théorique" (Ibid., p. 68) où, à travers l'amalgame du discours magique et de formules qui miment le discours scientifique, on peut retenir que le discours des manifestes exploite tous azimuts un langage qui vise à créer la fusion, l'osmose complète entre l'espace et le temps, le passé et l'avenir, l'ici et l'ailleurs, le dehors et le dedans. Ce mouvement est tout à fait à l'opposé de la science qui tend à 
86

séparer et non pas à unir. La "science" que défendent les manifestes est une idéologie (la contre-culture) et une phénoménologie, où la subjectivité se pare des clichés de l'objectivité scientifique. Cela dit, les positions défendues par les manifestes s'inscrivent dans un mouvement plus large qui éclaire les tensions au sein de l'institution littéraire de l'époque. En affirmant que le "poète n'est plus celui qui assume ou qui s'assume, mais bien celui qui travaille dans une chambre contiguë à celle du savant et du chercheur" (Infra, p. 23), ce n'est pas seulement le texte poétique québécois que Péloquin remet en question, c'est aussi le statut même de l'écrivain, ce qui rend la position de Péloquin contradictoire en regard de l'idéologie de la contre-culture. François Dumont lève la contradiction apparente en considérant que "[1]e discours initial de la contre-culture, du formalisme et de Claude Péloquin, malgré les divergences, constitue un même "moment de négation" [...]" ${ }^{40}$. Ainsi, la rupture avec le lyrisme, l'autobiographique et le discours national rapproche Péloquin de la poésie formaliste qui commence à s'imposer à la fin des années soixante. Les écrivains de La Barre du Jour se sont cherché aussi une légitimité axée sur une posture objective ou scientifique qui tablait sur les théories de leurs homologues de la revue Tel Quel, alors que les animateurs de la revue Quoi associent leur travail à celui du laboratoire. Chez Péloquin toutefois, ce n'est pas tant le texte comme l'homme qui devient le lieu de l'expérimentation: "Il y a parmi nous des hommes qui sont devenus leur propre laboratoire... en recherche sur un infiniment profond en eux-mêmes et sur leurs rayonnements." (Infra, p. 15). Les manifestes peuvent ainsi osciller sans transition entre les sciences occultes et les sciences de l'inconscient d'une part, et le vocabulaire des sciences pures d'autre part. Cette science caresse l'impossible rêve que le sujet et l'objet d'une même expérience puissent coïncider. Si cette phénoménologie récuse toute expression personnelle c'est en vertu de la prédominance de la perception sur les émotions. L'homme-objet auquel s'intéresse Péloquin

40. François Dumont, Usages de la poésie: le discours des poètes québécois sur la fonction de la poésie (1945-1970), Sainte-Foy, Presses de l'Université Laval/Créliq, 1993, p. 190, coll. "Vie des lettres québécoises". L'expression "moment de négation", pour qualifier l'opposition au passé qui définit la position idéologique des avant-gardes, est tirée de Jacques Pelletier, "Constitution d'une avant-garde littéraire", Études littéraires, vol. XX, $\mathrm{n}^{\circ} 1$, (printemps-été 1987), p. 111-130. 
est d'ailleurs essentiellement un univers physique, un espace dans lequel s'opèrent des mutations, des échanges dont on voudrait rendre compte tel qu'on le fait avec les molécules en chimie. L'homme est conçu simultanément comme l'objet de l'expérimentation, l'expérimentateur et le lieu de l'expérimentation, sorte de corps-laboratoire auquel il faut se mettre à l'écoute.

Par ailleurs, les textes de Péloquin présentent des affinités évidentes avec les manifestes futuristes. L'exaltation de la vie moderne, de la technologie et de la vitesse rappelle les prises de position esthétiques de Marinetti pour qui "une automobile rugissante, qui a l'air de courir sur de la mitraille, est plus belle que la Victoire de Samothrace" ${ }^{41}$. L'énergie, les ondes, le voyage, l'évacuation du Je introspectif, sont conformes à un discours qui reprend les attaques d'un discours nietzschéen contre l'homme faible, en l'occurrence la représentation du poète québécois transmise depuis Nelligan. "Ici, il n'y a pas que nos hivers, notre solitude de conquis, notre angoisse et nos souvenirs comme en témoigne presque toute notre littérature. Ici, il y a des ordinateurs, des autoroutes, des pylônes et de la technique." (Infra, p. 20). Dans la foulée des manifestes futuristes, des écrivains polonais ont signé à leur tour un texte intitulé les Primitivistes s'adressent aux nations du monde et à la Pologne, où est posée l'équivalence entre la science et les arts ("l'art est la science") et où on bannit l'usage du je psychologique. On ne peut être plus près du discours de Péloquin:

nous balayons de l'estaminet minable de l'infini des piètres créatures hystériques appelées poètes, accablées par l'insatisfaction, la douleur, la joie de vivre, l'extase, l'esthétique, l'inspiration, l'éternité.

Au lieu de l'esthétique l'anti-grâce, au lieu de l'extase - l'intellect. La création consciente et visant un but précis. ${ }^{42}$

Il n'est pas étonnant que de tels programmes tournent à la volonté technocratique d'imposer un programme en fonction du savoir nouveau dont on fait la promotion. Mais ce faisant, en privilégiant,

41. "Manifeste du futurisme" de Filippo Tomaso Marinetti, dans La poésie. Textes critiques $X I V^{e}-X X^{e}$ siècle, textes réunis par Jean-Marie Gleize, Paris, Larousse, 1995, p. 442. Le manifeste a paru pour la première fois dans les pages du Figaro en 1909. En caractères gras dans le texte.

42. Cité par Krysinski, loc. cit., p. 89. 
88

dans leur programme, la part consciente de la recherche, bien que l'objet lui-même soit le subconscient de l'artiste, les manifestes rompent avec l'un des paradigmes les plus puissants du Refus global, à savoir l'«intention, arme néfaste de la raison".

\section{Un programme technocratique}

Le but avoué est donc de gérer le savoir. "Subsiste croit fermement aux Pouvoirs qui unissent les êtres et les choses, c'està-dire à une gestion de l'Au-Dessus" (Subsiste, p. 143), lit-on dans le manifeste de 1965. On prône l'exclusion de ceux qui refusent de placer au sommet de leurs valeurs la recherche et l'art technicien pour en arriver à envisager des solutions aussi radicales que l'exil pur et simple des artistes qui refusent de se conformer au "règne de la recherche" (Infra, p.177). On lit encore: "Infra demande l'exil immédiat de tous ceux dont les ouvres ne dégagent rien d'insolite ou de fantastique" (Ibid., p.26). L'une des stratégies que partagent Péloquin et les tenants de la contre-culture qui viendront pas la suite, consiste dans le désir de s'emparer des moyens de production des médias électroniques pour les plier à leur programme: "Nous pensons sincèrement que, par les média d'influence, nous pouvons provoquer les mutations de l'Homme et du Cosmos." (Subsiste, p. 145). Le déni du politique est contredit par les prises de position à caractère socio-économique qui reçoivent quelques échos des luttes actuelles de nos jours :

Pourquoi, en médecine, par exemple, nos chercheurs se doivent-ils de réclamer à hauts cris des subsides accrus? Sinon de s'exiler comme l'ont fait les quelque deux mille chercheurs canadiens qui travaillent pour d'autres puissances. (Infra, p. 21).

Péloquin n'éprouve aucune méfiance envers la recherche institutionnelle, comme si elle échappait au politique, au contraire du milieu de l'édition, jugé retardataire. Il dénonce plutôt les limites présumément imposées à l'Université McGill et à la Société Radio-Canada: "quand est-ce que le centre d'électronique de l'université McGill va enfin servir à quelque chose! Les jeunes chercheurs en musique ont les mains liées. Quels seront ceux qui auront accès à ce centre de recherche visuelle que projette RadioCanada, si ce n'est des retardés?» (Infra, p. 22). Au nombre des demandes, qui défilent tout au cours des divers manifestes, retenons celles-ci, qui ont l'avantage de résumer l'orientation vers 
un programme aussi bien politique que scientifique et technocratique :

Expériences multipliées sur les maladies mentales, à tous les niveaux.

Multiplications de cliniques aux subsides accrus et formation de chercheurs [...].

Mise en chantier d'observatoires publics, pour visionner les profondeurs du ciel et de la terre.

Expérimentation sur la phénoménologie hallucinatoire en clinique et observation des réactions à différentes expériences sur des êtres de tous les niveaux d'équilibre.

Refonte du Service d'Aide à la Recherche au Ministère; (Infra, p.32).

Ce discours recouvre également des préoccupations en ce qui touche aux possibilités de se tailler une place dans le marché de l'édition et de la création artistique. Les revendications du groupe sont liées au désir, sur le plan socio-économique, de sortir de la marginalité. Le manifeste Infra condamne par exemple "l'aide absolument injustifiable accordée à des prétendus avantgardistes dans le domaine du spectacle" (Infra, p. 21). Et il demande "[à] qui va l'argent de tous les concours existants, si ce n'est à ceux qui ne risquent absolument rien?" (Ibid.). Le mode de répartition des avoirs (matériels) et des savoirs (symboliques) reste donc strictement à l'intérieur d'un espace idéologique favorable à l'expression artistique, sans pour autant remettre en question le pouvoir de l'État. Par le biais d'une recherche qui se voulait pluridisciplinaire, Péloquin et son groupe, au cours des années soixante, ont tenté de rompre avec une esthétique perçue comme monolithique; ils se situent donc à la fois dans la lignée du Refus global et dans la mouvance des courants formalistes qui émergent au cours de ces années. Mais leur poursuite d'une autonomie ne vise pas tant leur rapport au politique ou au religieux, comme ce fut le cas avec les automatistes ${ }^{43}$; l'engouement pour

43. Marcel Fournier écrit, dans une note infrapaginale: "[...] des artistes, dont Paul-Émile Borduas, refusent que le système de production de biens symboliques (art, littérature, etc.) soit dépendant du champ religieux et aussi du champ politique et revendiquent le droit de pouvoir définir eux-mêmes les normes de leur production et les critères d'évaluation de leurs produits." (L'entrée dans la modernité. Science, culture et société au Québec, Montréal, Éditions Saint-Martin, 1986, p. 158). Précisons que tout manifeste exige, à des degrés divers, le droit évoqué par Fournier. 
90

la science et la recherche institutionnelle permet aussi bien de rompre avec l'esthétique de l'Hexagone et de Parti pris que de donner une caution scientifique, dès lors soi-disant objective, aux esthétiques et aux idéologies post-surréaliste et contre-culturelle. 